\title{
STIFFNESS OF HARMONIC FUNCTIONS
}

\author{
CECILIA Y. WANG ${ }^{1}$
}

\begin{abstract}
Harmonic functions cannot change rapidly. For example, if $K$ is a compact subset of a Riemann surface $R$ and $\{u\}$ a family of harmonic functions $u$ on $R$ of nonconstant sign on $K$, then it is known that there exists a constant $q \in(0,1)$ independent of $u$ such that $\max _{K}|u|<q \sup _{R}|u|$ for all $u \in\{u\}$. In the present note we shall show that relations expressing such "stiffness" of harmonic functions can also be given for the Dirichlet norm and for the partial derivative with respect to the Green's function.
\end{abstract}

1. In the theory of harmonic functions, the significance of local distortion theorems, such as the above $q$-Lemma, lies in the fact that they are needed to establish the existence of global functions. Typically, Harnack's theorem, which gives the existence of a global harmonic function, is proved by Harnack's inequality, a local distortion theorem. Another example is the existence problem of the Evans-Selberg potential on arbitrary parabolic Riemann surfaces. This global problem was open for some three decades until M. Nakai [2] succeeded in solving it in the affirmative; one of the tools in his pioneering proof was the above local $q$-Lemma.

2. Let $R$ be a Riemann surface and $u$ a function on a set $F \subset R$. The Dirichlet integral of $u$ over $F$ is, by definition,

$$
D_{F}(u)=\int_{F}|\operatorname{grad} u|^{2} d x d y .
$$

Denote by $H(F)$ the class of harmonic functions on $F$. We shall show that the Dirichlet integral is restricted by the following distortion theorem:

THEOREM 1. For any compact subset $K$ of a Riemann surface $R$, there exists a constant $q_{D} \in(0,1)$ such that

$$
D_{K}(u) \leqslant q_{D} D_{R}(u)
$$

for all $u \in H(R)$.

Proof. Given any $z_{0} \in R$, it suffices to establish (1) for the class

$$
H_{1}(R)=\left\{u \in H(R) \mid D_{R}(u)=1, u\left(z_{0}\right)=0\right\}
$$

of functions. Suppose (1) is not true for $H_{1}(R)$. Then there exists a sequence $\left\{u_{n}\right\} \subset H_{1}(R)$ such that $D_{K}\left(u_{n}\right) \uparrow 1$. Since the $D_{R}\left(u_{n}\right)$ and $u_{n}\left(z_{0}\right)$ are bounded, $\left\{u_{n}\right\}$ is a normal family. By choosing a subsequence of $\left\{u_{n}\right\}$ if necessary, we

Received by the editors June 29, 1978.

AMS (MOS) subject classifications (1970). Primary 30A04.

${ }^{1}$ This work was sponsored by the Faculty Grant-in-Aid program, Arizona State University. 
may assume that $u_{n} \rightarrow u \in H(R)$ uniformly on compact subsets. Clearly $D_{K}(u)=1$ and $u \in H_{1}(R)$. This means that

$$
D_{R-K}(u)=\int_{R-K}|\operatorname{grad} u|^{2} d x d y=0 .
$$

Thus $|\operatorname{grad} u|^{2}=0$ on $R-K$, hence on $R$, and we have $D_{R}(u)=0$, a contradiction.

3. In addition to the Dirichlet integral of a harmonic function, it is also important to estimate the rate of growth of a harmonic function, for instance, in terms of directional derivatives. However, such derivatives are not defined on abstract Riemann surfaces, as they are not invariant under changes of local coordinates. The difficulty can be avoided by considering the relative rate of growth of a harmonic function with respect to a fixed reference function, e.g., the harmonic Green's function.

Let $\bar{R}$ be a compact bordered Riemann surface with border $\beta$. Fix a point $z_{0}$ in the interior $R$ of $\bar{R}$ such that the Green's function $g=g\left(\cdot, z_{0}\right)$ with pole $z_{0}$ satisfies $\operatorname{grad} g\left(\cdot, z_{0}\right) \neq 0$ on $\beta$.

TheOREM 2. Let $c \in(0, \infty)$ be a constant such that $\operatorname{grad} g\left(\cdot, z_{0}\right) \neq 0$ on the level line

$$
K_{c}=\left\{z \in R \mid g\left(z, z_{0}\right)=c, 0<c<\infty\right\}
$$

of $g$. Then there exists a constant $q_{0} \in(0,1)$ such that

$$
\max _{K_{c}}\left|\frac{\partial u}{\partial g}\right| \leqslant q_{0} \max _{\beta}\left|\frac{\partial u}{\partial g}\right|
$$

for all $u \in H(R) \cap C^{1}(\bar{R})$.

Proof. It suffices to consider nonconstant functions $u \in H(R) \cap C^{1}(\bar{R})$, as a constant $u$ would trivially give the equality in (2).

Let $v(z) \in H(R) \cap C(\bar{R})$ be the solution of the Dirichlet problem with boundary values $\partial u / \partial g$ on $\beta$. Consider the function

$$
w(z)=\int_{z_{0}}^{z} v(\zeta) d g\left(\zeta, z_{0}\right)
$$

Here the path of integration, determined by $d g^{*}={ }^{*} d g=0$ with $g^{*}$ the conjugate harmonic function of $g$, is an orthogonal trajectory of level curves of $g$. It corresponds to a radial line segment in the conformal image of $\bar{R}$ as a closed disk with radial slits whose edges have been suitably identified (cf. L. Sario and M. Nakai, Classification theory of Riemann surfaces, Springer, New York, 1969, p. 13). We must show that $w(z)$ is single-valued, or equivalently, that

$$
\int_{\gamma} v(\zeta) d g\left(\zeta, z_{0}\right)=0
$$

for every closed curve $\gamma$ through $z_{0} \in R$ on which $* d g=0$. By Poisson's 
formula,

$$
v(\zeta)=\frac{1}{2 \pi} \int_{\beta} v(z) * d g(z, \zeta)
$$

where

$$
* d g(z, \zeta)=\frac{\partial g(z, \zeta)}{\partial g\left(z, z_{0}\right)} * d g\left(z, z_{0}\right)
$$

Thus

$$
\int_{\gamma} v(\zeta) d g\left(\zeta, z_{0}\right)=\frac{1}{2 \pi} \int_{\beta} v(z)\left[\int_{\gamma} \frac{\partial g(z, \zeta)}{\partial g\left(z, z_{0}\right)} d g\left(\zeta, z_{0}\right)\right] * d g\left(z, z_{0}\right)
$$

To prove (4), it suffices to show that

$$
\frac{\partial g(z, \zeta)}{\partial g\left(z, z_{0}\right)} d g\left(\zeta, z_{0}\right) \equiv 0
$$

This in turn is true if

$$
s(z)=\int_{\gamma} g(z, \zeta) d g\left(\zeta, z_{0}\right) \equiv 0,
$$

a triviality for $z \in \beta$. Since $s$ is superharmonic on $R, s(z)=0$ on $\beta, s(z)>0$ on $R$, and $s\left(z_{0}\right)=0$, we conclude by the minimum principle that $s(z) \equiv 0$. We have shown that $w(z)$ is single-valued.

Next we prove $w(z) \in H(R)$. In view of the harmonicity of $v$, and the fact that the path of integration in (3) is determined by $d g^{*}\left(\cdot, z_{0}\right)=0$, we have

$$
\begin{aligned}
\frac{\partial^{2} w(z)}{\partial g^{*}\left(z, z_{0}\right)^{2}} & =\int_{z_{0}}^{z} \frac{\partial^{2} v(\zeta)}{\partial g^{*}\left(\zeta, z_{0}\right)^{2}} d g\left(\zeta, z_{0}\right) \\
& =\int_{z_{0}}^{z}-\frac{\partial^{2} v(\zeta)}{\partial g\left(\zeta, z_{0}\right)^{2}} d g\left(\zeta, z_{0}\right)=-\frac{\partial^{2} w(z)}{\partial g\left(z, z_{0}\right)^{2}}
\end{aligned}
$$

for all $z \in R-z_{0}$. Thus $\partial^{2} w / \partial g^{2}+\partial^{2} w / \partial g^{* 2}=0$, that is, $w$ is harmonic on $R-z_{0}$. By virtue of $v\left(z_{0}\right)=0, w(z)$ is bounded in a neighborhood of $z_{0}$, whence $z_{0}$ is a removable singularity and $w \in H(R)$.

Since $u-w \in H(R)$ and $\partial(u-w) / \partial g=v-v=0$ on $\beta$ we have $u=w$ + const and $\partial u / \partial g=\partial w / \partial g$ on $R$. By the defining property of $w(z), \partial w / \partial g$ $=v$, and therefore

$$
v=\frac{\partial u}{\partial g}
$$

on $R-z_{0}$.

To see that $v(z)$ is of nonconstant sign on $K_{c}$ let $\tilde{g}\left(\cdot, z_{0}\right)$ be the Green's function of the subregion of $R$ bounded by $K_{c}$. Then

$$
v\left(z_{0}\right)=\frac{1}{2 \pi} \int_{K_{c}} v(\zeta) * d \tilde{g}\left(\zeta, z_{0}\right)
$$


But

$$
\begin{aligned}
v\left(z_{0}\right) & =\frac{1}{2 \pi} \int_{\beta} v(\zeta) * d g\left(\zeta, z_{0}\right) \\
& =\frac{1}{2 \pi} \int_{\beta} \frac{\partial u}{\partial g} * d g\left(\zeta, z_{0}\right)=\frac{1}{2 \pi} \int_{\beta} * d u=0 .
\end{aligned}
$$

We conclude that $v(z)$ is not of constant sign on $K_{c}$. By the $q$-Lemma, there exists a $q_{0} \in(0,1)$ such that

$$
\max _{K_{c}}|v| \leqslant q \sup _{R}|v|=q \max _{\beta}|v| .
$$

In view of (5), our theorem follows.

4. For an illustration, take $\bar{R}=\{|z| \leqslant \rho\}$ with $\beta=\{z=\rho\}$. Here $g(z, 0)$ $=\log \rho r^{-1},|z|=r$, and $K_{c}=\left\{z|| z \mid=\rho e^{-c}\right\}$. For $u \in H(\mathrm{C}), \partial u / \partial g=$ - $r \partial u / \partial r$. By Theorem 2,

$$
\max _{K_{c}}\left|r \frac{\partial u}{\partial r}\right| \leqslant q_{0} \max _{\beta}\left|r \frac{\partial u}{\partial r}\right|,
$$

or

$$
\max _{K_{c}}\left|\frac{\partial u}{\partial r}\right| \leqslant q_{1} \max _{\beta}\left|\frac{\partial u}{\partial r}\right| .
$$

Thus our Theorem 2 complements Harnack's inequality on values of harmonic function by giving information on the rate of growth of harmonic functions.

\section{BIBLIOGRAPHY}

1. L. Ahlfors and L. Sario, Riemann surfaces, Princeton Univ. Press, Princeton, N. J., 1960.

2. M. Nakai, On Evans potential, Proc. Japan Acad. 38 (1962), 624-629.

3. K. Oikawa, A constant related to harmonic functions, Japan J. Math. 29 (1959), 111-113.

4. __ Sario's lemma on harmonic functions, Proc. Amer. Math. Soc. 11 (1960), 425-428.

5. B. Rodin and L. Sario, Convergence of normal operators, Kodai Math. Sem. Rep. 19 (1967), $165-173$.

6. L. Sario, A linear operator method on arbitrary Riemann surfaces, Trans. Amer. Math. Soc. 72 (1952), 281-295.

7. An integral equation and a general existence theorem for harmonic functions, Comment. Math. Helv. 38 (1964), 284-292.

8. __ Classification of locally Euclidean spaces, Nagoya Math. J. 25 (1965), 87-111.

9. L. Sario, M. Schiffer and M. Glasner, The span and principal functions in Riemannian spaces, J. Analyse Math. 15 (1965), 115-134.

10. L. Sario and G. Weill, Normal operators and uniformly elliptic partial differential equations, Trans. Amer. Math. Soc. 120 (1965), 225-235.

Department of Mathematics, Arizona State University, Tempe, Arizona 85281 